\title{
Ischaemic preconditioning for preventing contrast-induced AKI
}

The use of remote ischaemic preconditioning may prevent contrastmedium-induced acute kidney injury (AKI) in high-risk patients undergoing coronary angiography, according to the authors of a recent randomized, sham-controlled pilot study performed in Germany.

Contrast-induced AKI is a serious complication of coronary angiography and is associated with considerable morbidity and mortality. It is thought that the prevalence of this complication will increase owing to increasing use of contrast medium in diagnostic and interventional procedures. "We thought that contrast-induced AKI might partially be due to a worsening of renal microperfusion", states Fikret Er, an author on the paper. "Remote preconditioning has been associated with protection from ischaemic injury in various organs, and unpublished experimental data from our laboratory suggested that ischaemic preconditioning might be protective in ischaemic renal injury."

The latest study included 100 adults (mean age 73.2 years) with impaired renal function (serum creatinine $>124 \mu \mathrm{mol} / \mathrm{l}$ and/or estimated glomerular filtration rate $<60 \mathrm{ml} / \mathrm{min} / 1.73 \mathrm{~m}^{2}$ ) who were undergoing elective coronary angiography. Included patients were randomly assigned to either standard coronary angiography with sham preconditioning treatment prior to coronary angiography $(n=50)$ or to remote ischaemic preconditioning (through intermittent upper arm ischaemia using alternating inflations and deflations of a blood pressure cuff) prior to coronary angiography $(n=50)$.

\section{4 ...the procedure can be performed easily and virtually no safety concerns exist... 77}

The primary study outcome, AKI (defined as a serum creatinine increase of $>44 \mu \mathrm{mol} / \mathrm{l}$ or a relative increase of $\geq 25 \%$ from baseline within $48 \mathrm{~h}$ after exposure to contrast medium), occurred in significantly fewer patients in the ischaemic preconditioning group than in the control group (six patients [12\%] versus 20 patients [40\%], odds ratio in univariable analysis $0.21,95 \%$ CI $0.07-$ $0.57 ; P=0.002)$. No major adverse events related to the procedure were reported. "This very cheap and simple procedure seems to be very effective in lowering the incidence of contrast-induced AKI in high-risk patients with renal dysfunction", says Er. "Moreover, the procedure can be performed easily and virtually no safety concerns exist if performed appropriately."

Patients were followed up for 6 weeks after coronary angiography. During this time, the composite cardiovascular end point (death, rehospitalization or haemodialysis) occurred in significantly less of the preconditioning group than the control group (38\% versus $16 \% ; P=0.018$ ).

"The results of our trial are promising but limited to contrast-induced AKI and other surrogate parameters", states Er. "Data on morbidity and mortality are needed to establish this potentially protective regime in daily clinical settings. A second trial is planned to close this gap."

Rebecca Kelsey

Original article Er, F. et al. Ischemic preconditioning for prevention of contrast-medium-induced nephropathy: randomized pilot RenPro-Trial (Renal Protection Trial). Circulation doi:10.1161/CIRCULATIONAHA.112.096370 